\title{
Open-Access-Transformation in den Jüdischen Studien
}

Open Access für exzellente Publikationen aus den Jüdischen Studien: Dies ist das Ziel der gemeinsamen Initiative des Fachinformationsdiensts Jüdische Studien an der Universitätsbibliothek J. C. Senckenberg Frankfurt am Main und des Verlags Walter De Gruyter. Unterstützt von 18 Konsortialpartnern können 2020 insgesamt 8 Neuerscheinungen im Open Access Goldstandard veröffentlicht werden, darunter auch diese Publikation.

Die nachfolgenden wissenschaftlichen Einrichtungen haben sich an der Finanzierung beteiligt und fördern damit die Open-Access-Transformation in den Jüdischen Studien und gewährleisten die freie Verfügbarkeit für alle:

Fachinformationsdienst Jüdische Studien, Universitätsbibliothek J. C. Senckenberg

Frankfurt am Main

Staatsbibliothek zu Berlin - Preußischer Kulturbesitz

Universitätsbibliothek der Freien Universität Berlin

Universitätsbibliothek der Technischen Universität Berlin

Universitäts- und Landesbibliothek Düsseldorf

Universitätsbibliothek der Europa-Universität Viadrina Frankfurt (Oder)

Bibliothek der Vereinigten Theologischen Seminare der Georg-August-Universität Göttingen

Niedersächsische Staats- und Universitätsbibliothek Göttingen

Universitäts- und Landesbibliothek Sachsen-Anhalt

Staats- und Universitätsbibliothek Hamburg - Carl von Ossietzky

Gottfried Wilhelm Leibniz Bibliothek - Niedersächsische Landesbibliothek

Hochschule für Jüdische Studien Heidelberg

Universitäts- und Stadtbibliothek Köln

Universitätsbibliothek Mainz

Universitätsbibliothek der Ludwig-Maximilians-Universität München

Universitäts- und Landesbibliothek Münster

Herzog August Bibliothek Wolfenbüttel

Universitätsbibliothek Wuppertal 